\title{
REMARK ON THE SIMPLICIAL-COSIMPLICIAL TENSOR PRODUCT
}

\author{
RUDOLF FRITSCH
}

\begin{abstract}
We show that the existence of canonical representatives for the elements of the tensor product (coend) of a simplicial and a cosimplicial set depends only on the Eilenberg-Zilber property of the given cosimplicial set. Thus the second condition which is used in [5] for achieving this result is superfluous.
\end{abstract}

Let $X: \Delta^{\text {op }} \rightarrow S$ be a simplicial set and $Y: \Delta \rightarrow S$ a cosimplicial set. We consider $X$ as a $\mathbf{N}$-graded set with $\Delta$ acting on the right and correspondingly $Y$ is a $\mathbf{N}$-graded set with $\Delta$ acting on the left. The Eilenberg-Zilber Lemma states that every $x \in X$ has a unique decomposition

$$
x=x^{+} x^{\circ}
$$

with $x^{+}$nondegenerate and $x^{\circ}$ surjective. We assume that $Y$ has the dual property, i.e.

$$
\begin{gathered}
\text { every } y \in Y \text { has a tuique decomposition } \\
y=y^{+} y^{\circ} \\
\text { with } y^{+} \text {injective and } y^{\circ} \text { interior. }
\end{gathered}
$$

(That the proof of the Eilenberg-Zilber Lemma fails to be dualizable depends on the fact, that any surjective map in uniquely determined by the set of its sections; but different injective maps with the same one-element domain and the same codomain have the same set of retractions. Thus the Eilenberg-Zilber property for cosimplicial sets is a real restriction; see $[2,4.4$ and 4$]$ for a further discussion of this phenomenon.)

Now take

$$
T_{n}=\{(x, y) \mid x \in X, y \in Y, \text { degree } x=\text { degree } y=n\}
$$

for every $n \in \mathbf{N}$ and

$$
T=\coprod_{n \in \mathbf{N}} T_{n}
$$

thus $T$ also is a $\mathbf{N}$-graded set.

Received by the editors March 29, 1982.

1980 Mathematics Subject Classification. Primary 18G30.

Kev words and phrases. Simplicial set, cosimplicial set, tensor product, coend, Eilenberg-Zilber decomposition. 
A pair $(x, y) \in T$ is called similar to the pair $\left(x^{\prime}, y^{\prime}\right) \in T$ if there is an operator $\alpha \in \Delta$ such that

$$
x=x^{\prime} \alpha, \quad \alpha y=y^{\prime} .
$$

Similarity generates an equivalence relation $\sim$ on $T$ such that

$$
\left(x^{\prime} \alpha, y\right) \sim\left(x^{\prime}, \alpha y\right)
$$

for all suitable $\alpha$. The set of all equivalence classes is called the tensor product (coend [3]) of $X$ and $Y$.

A pair $(x, y) \in T$ is called minimal, if $x$ is nondegenerate and $y$ is interior. Our aim is to prove

THEOREM. Every equivalence class in T contains exactly one minimal element.

To this end we follow the lines of the proof in $[1,2.1]$ formulated for "subdivision" functors; see also [5]. We define maps $t_{1}, t_{r}, t: T \rightarrow T\left(M_{2}, M_{1}, M\right.$ in [5]) by taking

$$
\begin{aligned}
t_{r}(x, y) & =\left(x y^{+}, y^{\circ}\right), \\
t_{l}(x, y) & =\left(x^{+}, x^{\circ} y\right), \\
t & =t_{l} \circ t_{r} .
\end{aligned}
$$

Then clearly

$$
\begin{aligned}
(x, y) & \sim t_{l}(x, y) \sim t_{r}(x, y) \sim t(x, y) \\
t(x, y) & \neq(x, y) \Rightarrow \text { degree } t(x, y)<\operatorname{degree}(x, y), \\
t_{r}(x, y) & =(x, y) \Leftrightarrow y \text { interior, } \\
t_{l}(x, y) & =(x, y) \Leftrightarrow x \text { nondegenerate }
\end{aligned}
$$

and finally

$$
t(x, y)=(x, y) \Leftrightarrow(x, y) \text { minimal. }
$$

Since the set of degrees is bounded below, it follows from (11), that for any pair $(x, y) \in T$ the sequence

$$
\left(t^{n}(x, y)\right)_{n \in N}
$$

becomes stationary. Thus by (14) it contains a minimal pair, which by (10) is equivalent to the initial pair.

This proves the existence of a minimal pair in every class. The key to the uniqueness lies in the

LeMma. If $(x, y)$ is similar to $\left(x^{\prime}, y^{\prime}\right)$ then $t_{r}\left(x^{\prime}, y^{\prime}\right)$ is similar to $t(x, y)$.

This comes out by taking the operator

$$
\alpha^{\prime}=\left(x^{\prime}\left(\alpha y^{+}\right)^{+}\right)^{\circ}\left(\left(\alpha y^{+}\right)^{\circ} y^{\circ}\right)^{+}
$$

where the exponent ${ }^{+}$on an operator denotes its injective part while the exponent ${ }^{\circ}$ stands for the surjective component.

Now, for our goal it is enough to show that, if we start with two sequences (15) with similar pairs in $T$, we end up with the same minimal pair. So let the pair $(x, y)$ 
be similar to the pair $\left(x^{\prime}, y^{\prime}\right)$. The lemma provides us with the inductive argument for showing:

For all $n \in \mathrm{N}$ the pair $t_{r} t^{n}(x, y)$ is similar to the pair $t^{n}\left(x^{\prime}, y^{\prime}\right)$. But analogously as before, we have for sufficiently large $n$

$$
t_{r} t^{n}(x, y)=t^{n}(x, y)
$$

and both $t^{n}(x, y)$ and $t^{n}\left(x^{\prime}, y^{\prime}\right)$ are minimal pairs. In view of the equations (5) we see that in this case the corresponding $\alpha$ is injective and surjective, thus $\alpha=$ id, i.e.

$$
t^{n}(x, y)=t^{n}\left(x^{\prime}, y^{\prime}\right)
$$

which finishes the proof.

The reason for having written this note is that almost nobody seems to have read the German paper [1].

\section{BIBLIOGRAPHY}

I. R. Fritsch, Zur Unterteilung semisimplizialer Mengen. I, Math. Z. 108 (1969), 329-367.

2. R. Fritsch and D. M. Latch. Homotopv inverses for nerve, Math. Z. 177 (1981), 147-179.

3. S. Mac Lane, Categories. For the working mathematician, Springer-Verlag, Berlin and New York. 1972.

4. C. Ruiz and R. Ruiz, Remarks about the Eilenberg-Zilber type decomposition in cosimplicial sets, Rev. Colombiana Mat. 12 (1978), 61-82.

5. Characterization of the set-theoretical geometric realization in the noneuclidean case. Proc. Amer. Math. Soc. 81 (1981), 321-324.

Mathematisches Institut, LUdwig-Maximilians-Universitat, Theresienstrasse 39, D-8000 MUNCHEN, Federal Republic of Germany 